\title{
Beliefs about Tic Disorders and Tourette's Syndrome in South Korea: An Online Panel Survey
}

\author{
Minji Lee and Subin Park \\ Clinical Research Center, Mental Health Research Institute, National Center for Mental Health, Seoul, Korea
}

Objectives: This study investigates lay beliefs about the etiology and treatments of tic disorder and Tourette's syndrome, as well as identifying sociodemographic and personality variables affecting these beliefs among South Koreans.

Methods: In total, 673 participants (mean age $41.77 \pm 12.03$ years) completed an online survey regarding their beliefs about tic disorder and Tourette's syndrome. The factors related to their lay beliefs about the disorders were analyzed, and the correlates were investigated.

Results: Results indicated that lay people in South Korea held strong beliefs that the causes of tic disorder and Tourette's syndrome lie within the parenting/psychological and neurological/biological categories, compared to the dietary/environmental one. Among the sociodemographic variables, sex, age, and levels of subjective mental health knowledge were primarily associated with the aforementioned beliefs. Familiarity with tic disorder and Tourette's syndrome was also associated with these beliefs. Among the personality traits investigated, extraversion and conscientiousness had significant influences on the beliefs people had about tic disorder and Tourette's syndrome. Conclusion: The results suggest that both policy makers and mental health service providers should adopt a strategic approach for developing and implementing health education interventions about tic disorder and Tourette's syndrome because individual sociodemographic variables, familiarity with the disorders, and personality traits are all associated with the beliefs about these disorders.

Key Words: Tic disorders; Tourette syndrome; Beliefs; Personality; South Korea.

Received: April 11, 2019 / Revision: May 21, 2019 / Accepted: May 22, 2019

Address for correspondence: Subin Park, Clinical Research Center, Mental Health Research Institute, National Center for Mental Health, 127 Yongmasan-ro, Gwangjin-gu, Seoul 04933, Korea

Tel: +82-2-2204-0108, Fax: +82-2-2204-0280, E-mail: subin-21@hanmail.net

\section{INTRODUCTION}

Tics are defined as repetitive and involuntary motor movements and/or vocalizations that occur mainly during childhood and adolescence. Tics are typically seen as a transient condition, now referred to as "provisional tic disorder (TD)" in the Diagnostic and Statistical Manual of Mental Disorders, fifth edition (DSM-5) [1]. Simple and provisional TDs affect about 3-20\% of school-aged children [2]. When these symptoms persist for $\geq 1$ year, the condition is then referred to as "persistent TD," which has an estimated prevalence of 3-4\% among children [2]. Tourette's syndrome (TS), a clinical subtype of this disorder with the most severe and persistent tic symptoms, is characterized by multiple motor and at least one vocal tic that persist for at least 1 year [1]. TS was previously considered a rare entity, however, recent studies suggest a prevalence of approximately $1 \%$ among schoolaged children [3].

Despite many years of research, the underlying causes of

This is an Open Access article distributed under the terms of the Creative Commons Attribution Non-Commercial License (https://creativecommons.org/licenses/by-nc/4.0) which permits unrestricted non-commercial use, distribution, and reproduction in any medium, provided the original work is properly cited.
TD remain unclear. Previous etiological studies have suggested that neurobiological and genetic factors, as well as non-genetic and psychosocial factors, may have an interaction affecting the onset and severity of TD. Although studies indicate that non-genetic and psychosocial variables seem to affect the clinical manifestations of TD, and tend to modulate tic manifestations and the development of related symptoms [4], the results are inconsistent and related biological mechanisms are yet to be identified [5]. Conversely, neurobiological factors, such as basal ganglia dysfunctions and impaired functioning of the cortico-striato-thalamocortical circuits, have been consistently reported as related to TD development [2]. Therefore, the consensus is that neurobiological factors do play a major role in TD development. Recommended TD treatments, including behavioral interventions (such as habit reversal training, psychoeducation, and medication based therapies) that target the disorder's overall severity, have all been reported as equally effective [6].

Due to the negative attitudes towards TD, and various false beliefs about its causes, patients with this disorder are prone to experiencing social stigmatization and rejection [7], which in turn involves being harassed, bullied, and teased, resulting 
in an impaired quality of life [8]. Moreover, family members, especially the caregivers, of patients tend to internalize these negative, societal views and demonstrate both guilt and selfblame [8]. Considering the relatively high prevalence of TD among children, the unfounded beliefs about TD and TS, and the associated societal stigmatization, it is important to investigate factors related to lay peoples' beliefs or attitudes about these disorders. However, unlike other mental disorders, there is a lack of research on the beliefs or attitudes towards TD and TS.

Several sociodemographic variables, including sex [9], age [10], and education level [11,12], are associated with individual beliefs about mental illnesses. Moreover, familiarity with mental disorders has also been suggested as a factor affecting mental health knowledge and the beliefs about these forms of illnesses. For instance, personal (either with one's own diagnosis, or that of friends or relatives), work-related, or educational experiences with mental illnesses, as well as general familiarity with them, is significantly associated with improved mental health knowledge [13]. Furthermore, high school students having a family member affected by psychiatric problems are found to be more tolerant and exhibit greater positive attitudes towards people displaying TS symptoms [14].

Another possible variable affecting individual beliefs about mental illnesses is a person's unique personality traits. The broadly accepted Big-Five framework of personality traits classifies them into five broad domains: openness to experience, conscientiousness, extraversion, agreeableness, and neuroticism/emotional stability [15]. A recent study on the association between personality traits and overall health literacy found that neuroticism and extraversion increase the likelihood of a person possessing lower health literacy, whereas conscientiousness and openness to experience increased those odds among Americans aged over 50 years [16].

Although some studies have assessed either the public's knowledge about TS and its treatments [17] or their familiarity with and attitudes towards TD and TS [18], their samples were not representative of lay people, as they were primarily conducted on service providers and healthcare students. Moreover, to the best of our knowledge, no study has sought to identify the variables that may affect the public's beliefs on TD and TS. Therefore, we investigated public beliefs about the causes and treatment of TD and TS, and identified the relevant correlates. We specifically assessed the influence of sociodemographic factors, familiarity with the disorder, and personality traits on the beliefs about mental illnesses, based on the results of previous studies [14,16].

\section{METHODS}

\section{Participants}

We conducted an online survey of South Korean residents aged from 20-64 years. It was created by an online survey service provider, which operates using a consumer panel (www. invight.co.kr) with numerous potential respondents. Questionnaires were sent randomly, via e-mail, to 12976 registered and non-duplicated potential respondents who had participated in a survey at least once within the preceding year. Proportionate allocation, according to population age distributions in South Korea, was utilized. Informed consent was obtained from each participant, and the survey did not contain any personally identifiable questions. In total, 673 respondents (337 men and 336 women; mean age, $41.77 \pm 12.03$ years) participated in this survey and successfully completed the questionnaire. This research was reviewed and approved by the Institutional Review Board of the National Center for Mental Health (No. 116271-2017-38).

\section{Measurements}

\section{Sociodemographic data}

Sociodemographic data pertaining to participants' age, sex, family income status, education level, and religion were collected. Regarding personal familiarity with psychiatric disorders, respondents were asked whether they or any of their family members, relatives, or friends have TD or TS.

\section{Subjective mental health knowledge}

Subjective mental health knowledge levels were self-rated by the respondents on a 7-point Likert scale (1 indicating "I know nothing" and 7 indicating "I know a lot") based on the question: "How much do you think you know about psychiatric disorders?"

\section{Personality}

A Ten-Item Personality Inventory (TIPI) $[19,20]$ was used to assess the participants' personality traits. TIPI is a brief measure of the Big Five personality dimensions. Ten items were scored, each on a 7-point Likert scale (1 indicating "disagree strongly" and 7 indicating "agree strongly"). The individual levels of the five personality characteristics of each respondent were determined according to their final TIPI scale score.

\section{Beliefs about TD and TS}

A description of TD and TS, based on the DSM- 5 criteria, was provided to the participants, who were then asked to rate their agreement or disagreement with various statements relating to beliefs about these disorders. The statements were 
adapted from the TS survey [17], originally designed to assess individuals' knowledge regarding this disorder, as well from the attention-deficit/hyperactivity disorder (ADHD) beliefs scale [21], which measures parents' beliefs and attitudes regarding ADHD. A professional team, consisting of a pediatric psychiatrist and a clinical psychologist, selected, translated, and revised the items for use by the South Korean lay population. All of the selected items were categorized into three categories: parenting/psychological beliefs (11 items), neurological/biological beliefs (8 items), and dietary/environmental beliefs ( 7 items). The respondents were asked to rate the extent of either their agreement or disagreement with each of the given statements on a 7-point Likert scale (1 representing "disagree strongly" and 7 representing "agree strongly").

\section{Statistical analysis}

Descriptive statistics (mean \pm standard deviation) were calculated in order to describe the basic characteristics of the data. Reliability analysis was conducted to measure the internal consistency of each category of the TS beliefs scale. Multiple linear regression analysis was performed to assess the potential associations between the participants' sociodemographic characteristics, their familiarity with TD and TS, subjective knowledge about psychiatric disorders, and personality variables with their beliefs about TD and TS. Total scores for each of the categories of the TD and TS beliefs were entered as the dependent variables. Variables including sex (men indicated by 0 and women indicated by 1 ), age, family income (per 1000 USD), religious status ("no" shown by 0 and "yes" by 1), education level, subjective mental health knowledge, familiarity with TD and TS ("no" shown by 0 and "yes" by 1), and the five personality variables were entered simultaneously as the independent variables. SPSS Statistics version 20 (IBM Corp., Armonk, NY, USA) was used for all the statistical analyses conducted. For the corrections due to the occurrence of multiple testing, statistical significance was specified using a Bonferroni correction. Therefore, a pvalue of $<0.0167$ (which $=0.05 / 3$ outcome variables) was considered as statistically significant.

\section{RESULTS}

Table 1 contains the sociodemographic characteristics of the 673 participants $-50.1 \%$ of the participants were men, their average age was $41.77 \pm 12.03$ years, $56.7 \%$ claimed to be university graduates, and approximately $49.5 \%$ indicated that they held to no religion. There was no significant difference in the age distribution according to sex $(\mathrm{p}=0.809)$. With respect to the familiarity with TD and TS, $13.5 \%$ of partici-
Table 1. Demographic characteristics of the study population

\begin{tabular}{|c|c|}
\hline Variable & $\mathrm{N}(\%)$ \\
\hline Sex, men & $337(50.1)$ \\
\hline Age, years, mean $\pm S D$ & $41.77 \pm 12.03$ \\
\hline \multicolumn{2}{|l|}{ Income, \$ } \\
\hline$<2000$ & $78(11.6)$ \\
\hline $2000-2990$ & $99(14.7)$ \\
\hline $3000-3990$ & 130 (19.3) \\
\hline $4000-4990$ & 107 (15.9) \\
\hline $5000-5990$ & 107 (15.9) \\
\hline $6000-6990$ & $62(9.2)$ \\
\hline$>7000$ & $90(13.4)$ \\
\hline \multicolumn{2}{|l|}{ Education } \\
\hline Under high school & $95(14.1)$ \\
\hline College & $112(16.6)$ \\
\hline University & $382(56.7)$ \\
\hline Graduate school & $84(12.5)$ \\
\hline \multicolumn{2}{|l|}{ Religion } \\
\hline None & $333(49.5)$ \\
\hline Protestant & $163(24.2)$ \\
\hline Roman Catholic & $80(11.9)$ \\
\hline Buddhism & $96(14.3)$ \\
\hline Others & $1(0.1)$ \\
\hline Having TD/TS & $91(13.5)$ \\
\hline Oneself & $4(0.6)$ \\
\hline Family & $14(2.1)$ \\
\hline Relatives & $11(1.6)$ \\
\hline Friends & $62(9.2)$ \\
\hline
\end{tabular}

pants knew someone who either had or has one of these disorders. As only four of the participants claimed to have been diagnosed with TD or TS, the levels of familiarity with these disorders was not separated by response types (i.e., whether they or their family members/friends had TD or TS), and was used as a single variable in the analysis as a result. The mean subjective mental health knowledge level was 3.76 \pm 1.23 .

Table 2 presents the mean and standard deviations for each item and category, as well as the internal consistency among the categories themselves. In the comparison of the mean scores for each category, the scores for parenting/psychology were highest (4.50), followed by those for neurological/biological (4.25) and dietary/environmental (3.88). Among all of the questionnaire items, the participants reported the strongest beliefs for behavior therapy as an effective treatment for TS (5.24 \pm 1.13$)$, followed by the belief that TS is a neurological disorder (5.18 \pm 1.11$)$. Among the beliefs about the causes and treatments of TS, the top 10 items with the highest mean scores were included in the parenting/psychology or neurological/biological categories. Each category showed high in- 
Table 2. Beliefs about the causes and treatment of TS

\begin{tabular}{|c|c|c|}
\hline Categories/items & Mean (SD) & Cronbach's alpha \\
\hline Parenting and psychological & 4.50 & 0.799 \\
\hline Behavior therapy (relaxation training, habit reversal training) is an effective treatment for TS & $5.24(1.13)$ & \\
\hline Psychological counseling is an effective treatment for TS & $4.90(1.21)$ & \\
\hline TS is caused by family problems, such as alcoholism or marital discord & $4.77(1.30)$ & \\
\hline Using special parenting techniques is an effective way of treating TS & $4.63(1.28)$ & \\
\hline Stresses, such as schooling or friendship, are the cause of TS & $4.62(1.21)$ & \\
\hline Tics get worse as they are being discussed with the TS patient & $4.59(1.24)$ & \\
\hline It would be better for parents to show an attitude that they do not care about the tic itself & $4.56(1.40)$ & \\
\hline TS is caused by ineffective discipline & $4.35(1.35)$ & \\
\hline People with TS can suppress their tics & $3.82(1.42)$ & \\
\hline Children develop TS because they want attention & $3.70(1.48)$ & \\
\hline Intentionally suppressing tics causes them to be worse or causes another tic to occur & $3.33(1.40)$ & \\
\hline Neuro and biological & 4.25 & 0.826 \\
\hline TS is a neurological disorder & $5.18(1.11)$ & \\
\hline Medication of TS is effective by changing the neurotransmitter in the brain & $4.66(1.12)$ & \\
\hline I would not hesitate to offer medication to children with TS if a doctor recommends it & $4.48(1.38)$ & \\
\hline Medication is a safe treatment for TS & $4.44(1.38)$ & \\
\hline Medication is almost always effective in treating TS & $4.16(1.16)$ & \\
\hline TS can be caused by complications during pregnancy & $3.99(1.22)$ & \\
\hline The only treatment for TS is medication & $3.74(1.42)$ & \\
\hline TS is genetically transmitted & $3.33(1.40)$ & \\
\hline Dietary and environmental & 3.88 & 0.839 \\
\hline Special diets are effective treatments for TS & $4.23(1.32)$ & \\
\hline Overexposure to TV/internet/smartphone is the cause of TS & $4.11(1.35)$ & \\
\hline TS is caused by exposure to environmental substances, such as lead & $4.07(1.24)$ & \\
\hline Reducing a child's sugar intake is an effective treatment for TS & $3.99(1.18)$ & \\
\hline Vitamin therapy is an effective treatment for TS & $3.95(1.13)$ & \\
\hline TS is caused by allergic reactions or sensitivity to food preservatives & $3.42(1.33)$ & \\
\hline Bacterial infection is the cause of TS & $3.40(1.35)$ & \\
\hline
\end{tabular}

SD: standard deviation, TS: Tourette's syndrome

ternal consistency (parenting/psychological category: 0.799, neurological/biological category: 0.826 , and dietary/environmental category: 0.839).

Table 3 describes the observed associations between participants' sociodemographic characteristics, their familiarity with TD and TS, and their personality traits and beliefs about TD and TS. Women as well as those with higher subjective mental health knowledge, reported greater beliefs in the parenting/psychological category. Participants who had higher subjective mental health knowledge and higher scores for the conscientiousness personality trait showed stronger beliefs in the neurological/biological category. Older respondents, extroverts, and participants who were less familiar with TD and TS (either having never been diagnosed or without any acquaintances with TD or TS) reported stronger beliefs in the dietary/environmental category.

\section{DISCUSSION}

This study aimed to examine lay beliefs about TD and TS, in particular regarding its causes and treatments, and then to assess the influence of sociodemographic variables, familiarity levels, and personality traits on these beliefs. The main findings and their implications are as follows.

Compared with their male counterparts, the female participants tended to have stronger beliefs in the parenting/psychological category for TD and TS causation, whereas no sexbased differences were observed in the neurological/biological and dietary/environmental categories. In South Korea, women tend to be more involved and interested in the process of childcare when compared to men [22]. Taking into consideration that the caregivers of children with TD or TS tend to experience feelings of guilt or self-blame [8], efforts should be 
Table 3. Results of regression analysis of beliefs about TS

\begin{tabular}{|c|c|c|c|c|c|c|c|c|c|}
\hline \multirow{2}{*}{ Predictors } & \multicolumn{3}{|c|}{ Parenting and psychological } & \multicolumn{3}{|c|}{ Neuro and biological } & \multicolumn{3}{|c|}{ Dietary and environmental } \\
\hline & $\mathrm{B}$ & $t$ & $\mathrm{P}$ & $B$ & $t$ & $p$ & $B$ & $t$ & $p$ \\
\hline Sex (men, 0; women, 1) & 1.685 & 2.712 & 0.007 & 0.046 & 0.088 & 0.930 & 0.752 & 1.537 & 0.125 \\
\hline Age (years) & 0.058 & 2.129 & 0.034 & 0.005 & 0.206 & 0.837 & 0.063 & 2.938 & 0.003 \\
\hline Income & 0.209 & 1.501 & 0.134 & 0.039 & 0.325 & 0.746 & -0.069 & -0.631 & 0.528 \\
\hline Religion (no, 0; yes, 1) & 0.972 & 1.549 & 0.122 & -0.180 & -0.337 & 0.736 & 0.366 & 0.740 & 0.459 \\
\hline Education & -0.040 & -0.219 & 0.827 & -0.116 & -0.738 & 0.461 & -0.032 & -0.223 & 0.824 \\
\hline Subjective mental health knowledge level & 0.960 & 3.671 & 0.000 & 1.055 & 4.732 & 0.000 & 0.422 & 2.047 & 0.041 \\
\hline Tic/TS (no, 0; yes, 1) & 1.268 & 1.420 & 0.156 & -0.885 & -1.163 & 0.245 & -2.261 & -3.214 & 0.001 \\
\hline Extraversion & 0.315 & 2.251 & 0.025 & -0.057 & -0.481 & 0.631 & 0.323 & 2.935 & 0.003 \\
\hline Agreeableness & 0.280 & 1.481 & 0.139 & -0.001 & -0.004 & 0.997 & 0.043 & 0.291 & 0.771 \\
\hline Conscientiousness & 0.187 & 1.143 & 0.254 & 0.472 & 3.378 & 0.001 & -0.077 & -0.594 & 0.553 \\
\hline Emotional stability & -0.178 & -1.097 & 0.273 & -0.186 & -1.344 & 0.180 & 0.018 & 0.144 & 0.885 \\
\hline Openness to experience & -0.374 & -2.160 & 0.031 & -0.149 & -1.009 & 0.313 & -0.283 & -2.075 & 0.038 \\
\hline$F(12660)$ & 5.246 & & 0.000 & 3.403 & & 0.000 & 3.624 & & 0.000 \\
\hline Adjusted $\mathrm{R}^{2}$ & 0.070 & & & 0.041 & & & 0.045 & & \\
\hline
\end{tabular}

All variables are concurrently entered in multiple linear analyses. B: unstandardized coefficient, TS: Tourette's syndrome

made to correct this particular misbelief among women that these disorders are due to improper parenting.

Older respondents tended to hold stronger beliefs about the etiology of TD and TS in the dietary/environmental category, which is in line with the findings of previous studies. Older individuals generally share similar beliefs about mental illnesses, and an overall willingness to seek help, with younger adults [10]. However, they also tend to hold more untested beliefs about these disorders than the younger population [23]. Moreover, considering that older age is positively associated with negative attitudes towards mental disorders [24], this result stresses the importance of proper education in correcting the untested beliefs of older adults.

Previous studies have suggested that views on mental disorders tend to vary with peoples' education levels $[11,12]$. However, no significant influence due to participants' education level was observed in this study. This inconsistency may be attributable to the upward mobility of the education levels in South Korea, with the university enrollment rate being $>80 \%$ nationally [25]. In addition, as this study utilized an online survey of internet users, the sample may be biased towards a higher education group.

Subjective mental health knowledge level of each participant was significantly associated with stronger beliefs about TD and TS in the parenting/psychological and neurological/ biological categories. This result shows that those who are confident in their mental health knowledge have relatively accurate and up-to-date knowledge about TD and TS compared to those who are not as confident in this area.

Respondents who had experience with TD or TS (involving either the self or a close acquaintance) were more likely to hold lower beliefs in the dietary/environmental category, suggesting that familiarity with these disorders facilitates a better understanding of their true etiologies, and instills correct beliefs about the disorders. This finding is consistent with previous studies, which showed that people tend to have better mental health knowledge if they are familiar with mental illnesses [14]. However, due to the low prevalence of TD and TS (especially among adults, where it is $0.1-1 \%$ ) [3], the general population may not be familiar with these diseases. Therefore, concerted efforts on raising public awareness about these diseases are essential.

Among the five analyzed personality traits, extroversion was related to beliefs in the dietary/environmental etiological category, with conscientiousness being related to beliefs in the neurological/biological one. These results are consistent with those of a previous study on health literacy conducted among adults aged $>50$ years [16]. In this study, extroverts tended to have lower health literacy, whereas conscientious people tended to possess higher health literacy (i.e., correctly recognizing medical terms) [16]. The main feature of extroversion is the tendency to engage in and enjoy social attention, and to pursue excitement, novelty, and challenges. This can be interpreted as an open attitude towards various external stimuli, including previously untried ones [26]. Furthermore, it has been suggested that extroverts show lower retention of learned knowledge [27], which constrains their overall retention of correct information related to mental health. Conscientiousness is characterized by both deliberation and compliance with rules and norms. Therefore, people with higher conscientiousness are more likely to be careful, thorough, organized, and rational [26]. Conscientiousness might, as a re- 
sult, elevate a person's health literacy through proper health service usage and self-education [16]. These results imply that a person's personality traits are a strong predictor of their mental health literacy. Therefore, considering individual differences in personality traits during the provisioning of related services is important.

This study has various limitations. First, the sample may have been biased towards a higher education group. The responses might also be biased, as this study utilized a self-reported survey of online users. Moreover, only potential associations between variables could be revealed due to the design of this study (i.e., cross-sectional). Nevertheless, as the age distribution of the sample mirrored that of the adults in the general South Korean population, it can be considered as substantially representative of lay people in this country. A longitudinal study, with a representative sample, needs to be conducted in the future as an extension of this research. Comparative studies between research conducted in different countries would also help to identify the similarities and differences in beliefs regarding the causes and treatments of TD and TS, and their correlates.

\section{CONCLUSIONS}

This study revealed that lay people in South Korea tend to hold relatively reasonable beliefs about the etiology and treatment of TD and TS. Here, parenting/psychological and neurological/biological beliefs tended to be more prevalent than dietary/environmental ones. We also found that sociodemographic characteristics (such as sex and age), subjective mental health knowledge levels, familiarity, and the Big Five personality traits (such as extraversion and conscientiousness) influence peoples' beliefs about TD and TS.

Our findings provide useful information for mental health service providers and policy makers who oversee public education about mental illnesses. It can be seen that sociodemographic characteristics should be considered when providing educational services. Those who are women, older, and are not familiar with TD and TS should be the first targets to approach and be provided with accurate information about these disorders. In addition to individual sociodemographic differences and experiences with these disorders, inter-individual personality differences should be considered in the promotion of public literacy about TD and TS. Moreover, as brief education about these disorders is less likely to induce a significant change [28], and personality traits are not easily modified [29], it is crucial to adopt a strategic approach for delivering accurate information about TD and TS.

\section{Acknowledgments}

This work was supported by a clinical research grant (No. 2019-03) from the National Center for Mental Health, Republic of Korea.

\section{Conflicts of Interest}

The authors have no potential conflicts of interest to disclose.

\section{REFERENCES}

1) American Psychiatric Association. Diagnostic and statistical manual of mental disorders (DSM- $5^{\circledR}$ ). Washington, DC: American Psychiatric Pub;2013.

2) Tagwerker Gloor F, Walitza S. Tic disorders and Tourette syndrome: current concepts of etiology and treatment in children and adolescents. Neuropediatrics 2016;47:84-96.

3) Kadesjö B, Gillberg C. Tourette's disorder: epidemiology and comorbidity in primary school children. J Am Acad Child Adolesc Psychiatry 2000;39:548-555.

4) Hoekstra PJ, Dietrich A, Edwards MJ, Elamin I, Martino D. Environmental factors in Tourette syndrome. Neurosci Biobehav Rev 2013; 37:1040-1049.

5) Chao TK, Hu J, Pringsheim T. Prenatal risk factors for Tourette syndrome: a systematic review. BMC Pregnancy Childbirth 2014;14:53.

6) Mills S, Hedderly T. A guide to childhood motor stereotypies, tic disorders and the tourette spectrum for the primary care practitioner. Ulster Med J 2014;83:22-30.

7) Marcks BA, Berlin KS, Woods DW, Davies WH. Impact of Tourette syndrome: a preliminary investigation of the effects of disclosure on peer perceptions and social functioning. Psychiatry 2007;70:5967.

8) Rivera-Navarro J, Cubo E, Almazán J. The diagnosis of Tourette's syndrome: communication and impact. Clin Child Psychol Psychiatry 2009;14:13-23.

9) Bener A, Ghuloum S. Gender differences in the knowledge, attitude and practice towards mental health illness in a rapidly developing Arab society. Int J Soc Psychiatry 2011;57:480-486.

10) Segal DL, Coolidge FL, Mincic MS, O'Riley A. Beliefs about mental illness and willingness to seek help: a cross-sectional study. Aging Ment Health 2005;9:363-367.

11) Hamid A, Furnham A. Factors affecting attitude towards seeking professional help for mental illness: a UK Arab perspective. Ment Health Relig Cult 2013;16:741-758.

12) Lauber C, Falcato L, Nordt C, Rössler W. Lay beliefs about causes of depression. Acta Psychiatr Scand Suppl 2003;108:96-99.

13) Hansson L, Stjernswärd S, Svensson B. Changes in attitudes, intended behaviour, and mental health literacy in the Swedish population 2009-2014: an evaluation of a national antistigma programme. Acta Psychiatr Scand 2016;134 Suppl 446:71-79.

14) Brook U, Boaz M. Attitude and knowledge of high school pupils towards adolescents with special needs (Tourette's syndrome). Indian J Pediatr 2006;73:1099-1104.

15) Goldberg LR. An alternative "description of personality": the bigfive factor structure. J Pers Soc Psychol 1990;59:1216-1229.

16) Kim H, Zhang Q, Svynarenko R. Personality traits and health literacy. Journal of Family Medicine \& Community Health 2017;4:11021108.

17) Marcks BA, Woods DW, Teng EJ, Twohig MP. What do those who know, know? Investigating providers' knowledge about Tourette's syndrome and its treatment. Cogn Behav Pract 2004;11:298-305.

18) Katona C. Familiarity with and attitudes to Tourette's syndrome in healthcare students: a pilot comparison with epilepsy. Eur J Psychiatry 2013;27:129-136.

19) Gosling SD, Rentfrow PJ, Swann WB. A very brief measure of the big-five personality domains. J Res Pers 2003;37:504-528.

20) Lee M, Sul S, Kim H. The impact of moral decision style on impres- 
sion formation. Korean Journal of Social and Personality Psychology 2014;28:201-223.

21) Johnston C, Freeman W. Parents' beliefs about ADHD: implications for assessment and treatment. The ADHD Report 2002;10:6-9.

22) Oh H, Lee EK. Caregiver burden and social support among mothers raising children with developmental disabilities in South Korea. International Journal of Disability, Development and Education 2009; 56:149-167.

23) Farrer L, Leach L, Griffiths KM, Christensen H, Jorm AF. Age differences in mental health literacy. BMC Public Health 2008;8:125.

24) Dietrich S, Beck M, Bujantugs B, Kenzine D, Matschinger H, Angermeyer MC. The relationship between public causal beliefs and social distance toward mentally ill people. Aust N Z J Psychiatry 2004;38:348-354.
25) Kim T. A study on structure changes of university based on data for 15 years. Journal of The Korean Data Analysis Society 2014;16:187200.

26) Roccas S, Sagiv L, Schwartz SH, Knafo A. The big five personality factors and personal values. Pers Soc Psychol Bull 2002;28:789-801.

27) Arbuckle TY, Gold D, Andres D. Cognitive functioning of older people in relation to social and personality variables. Psychol Aging 1986;1:55-62.

28) Friedrich S, Morgan SB, Devine C. Children's attitudes and behavioral intentions toward a peer with Tourette syndrome. J Pediatr Psychol 1996;21:307-319.

29) McCrae RR, Costa Jr PT, Martin TA, Oryol VE, Senin IG, O'Cleirigh C. Personality correlates of HIV stigmatization in Russia and the United States. J Res Pers 2007;41:190-196. 